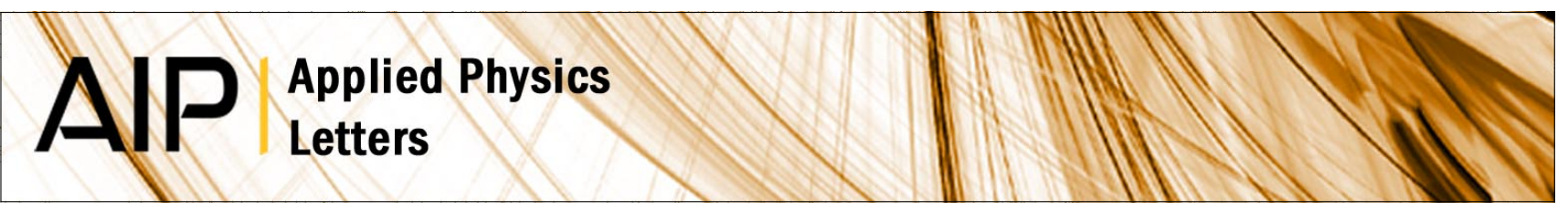

\title{
Angular emission properties of a layer of rare-earth based nanophosphors embedded in one-dimensional photonic crystal coatings
}

O. Sánchez-Sobrado, A. M. Yacomotti, M. E. Calvo, O. E. Martínez, M. Ocaña et al.

Citation: Appl. Phys. Lett. 99, 051111 (2011); doi: 10.1063/1.3619814

View online: http://dx.doi.org/10.1063/1.3619814

View Table of Contents: http://apl.aip.org/resource/1/APPLAB/v99/i5

Published by the American Institute of Physics.

\section{Related Articles}

Multiwave out-of-normal band-edge lasing in cholesteric liquid crystals

J. Appl. Phys. 112, 013105 (2012)

Mesoporous silicon photonic structures with thousands of periods

J. Appl. Phys. 112, 013106 (2012)

In situ optofluidic control of reconfigurable photonic crystal cavities

Appl. Phys. Lett. 100, 261107 (2012)

Pattern-integrated interference lithography instrumentation

Rev. Sci. Instrum. 83, 063707 (2012)

All-optical tuning of a quantum dot in a coupled cavity system

Appl. Phys. Lett. 100, 231107 (2012)

\section{Additional information on Appl. Phys. Lett.}

Journal Homepage: http://apl.aip.org/

Journal Information: http://apl.aip.org/about/about_the_journal

Top downloads: http://apl.aip.org/features/most_downloaded

Information for Authors: http://apl.aip.org/authors

\section{ADVERTISEMENT}

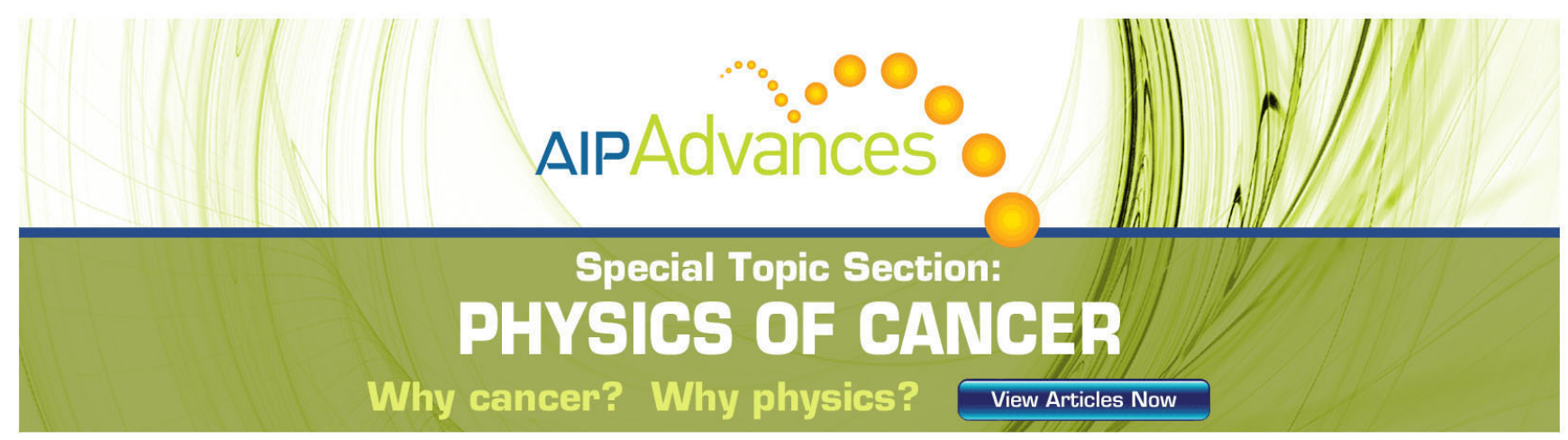




\title{
Angular emission properties of a layer of rare-earth based nanophosphors embedded in one-dimensional photonic crystal coatings
}

\author{
O. Sánchez-Sobrado, ${ }^{1}$ A. M. Yacomotti, ${ }^{2}$ M. E. Calvo, ${ }^{1}$ O. E. Martínez, ${ }^{3}$ M. Ocaña,${ }^{1}$ \\ N. Núñez, ${ }^{1}$ J. A. Levenson, ${ }^{2}$ and H. Míguez ${ }^{1, a)}$ \\ ${ }^{1}$ Instituto de Ciencia de Materiales de Sevilla (CSIC-US), C/Américo Vespucio, 49, 41092 Sevilla, Spain \\ ${ }^{2}$ Laboratoire de Photonique et de Nanostructures (CNRS), Marcoussis, Route de Nozay, 91460 Marcoussis, \\ France \\ ${ }^{3}$ Universidad de Buenos Aires, Intendente Güiraldes, 2160, Pabellón I, Ciudad Universitaria, Buenos Aires \\ C1428EHA, Argentina
}

(Received 4 March 2011; accepted 1 July 2011; published online 4 August 2011)

\begin{abstract}
The angular properties of light emitted from rare-earth based nanophosphors embedded in optical resonators built in one-dimensional photonic crystal coatings are herein investigated. Strong directional dependence of the photoluminescence spectra is found. Abrupt angular variations of the enhancement caused by the photonic structure and the extraction power are observed, in good agreement with calculated polar emission patterns. Our results confirm that the optical cavity favors the extraction of different wavelengths at different angles and that integration of nanophosphors within photonic crystals provides control over the directional emission properties that could be put into practice in phosphorescent displays. (c) 2011 American Institute of Physics. [doi:10.1063/1.3619814]
\end{abstract}

In recent years, multiple applications for rare-earth based nanoparticles, usually known as nanophosphors, have been developed in several fields like light emitting diodes (LEDs) ${ }^{1}$ sensors, ${ }^{2}$ and displays. ${ }^{3}$ In the latter case, all research efforts have been focused on the improvement of the photoluminescence efficiency by changing the conditions of synthesis. ${ }^{4-9}$ However, in a work recently reported by some of us, an important enhancement of the photoluminescence of Eu-doped yttrium fluoride colloidal particles was obtained by embedding them in a resonator built in a one dimensional photonic crystal (1DPC). ${ }^{10}$ By matching the spectral position of the cavity resonance with that of a specific luminescent band, strong reinforcement of its intensity was observed, while the rest of the bands were dramatically attenuated. These phenomena occurred along the direction perpendicular to the multilayer surface. On the other hand, due to their ability to tailor the angular characteristics of emission, photonic crystals have been widely used to improve the performance of several photonic devices as biosensors ${ }^{11}$ and LEDs. ${ }^{12,13}$ As far as we know, there are no previous reports on the directional emission of nanophosphors integrated in photonic crystals, which might be of relevance for their potential application in displays.

In this work, we study experimentally the variation of the intensity of the photoluminescence bands of optical resonators containing nanophosphors as the angle of collection is changed. We observe a strong angular dependence of the spectrally selective enhancement achieved with respect to reference samples, which is in turn largely affected by the values of the optical parameters of the resonator. Our experimental data are analyzed on the basis of a simple theoretical model proposed by Benisty et al., ${ }^{14}$ a qualitative agreement being found for the directional properties of both the extracted intensity and the enhancement of selected emission lines.

${ }^{a}$ Electronic mail: hernan@icmse.csic.es.
Samples were prepared following a procedure reported elsewhere. ${ }^{10}$ Briefly, the optical resonator was built by sandwiching a layer of Eu-doped yttrium fluoride nanophosphors ${ }^{15}$ between two 1DPC consisting in alternated layers of $\mathrm{SiO}_{2}$ and $\mathrm{TiO}_{2}$ nanoparticles. ${ }^{16}$ The nanophosphor layer was surrounded by two $\mathrm{SiO}_{2}$ nanoparticle multilayers that disrupted the periodicity of the whole ensemble, leading to the opening of resonant modes. The nanophosphors employed emit photoluminescent bands in the visible range whose width $\Delta \lambda_{\mathrm{B}}$ is narrower than that of the cavity resonance, $\Delta \lambda_{\mathrm{c}}\left(\Delta \lambda_{\mathrm{c}} / \Delta \lambda_{\mathrm{B}} \cong 6\right)$. Following a spin coating protocol, ${ }^{17}$ the resonator can be devised to present the resonance at the desired spectral position. The reference is prepared by sandwiching, between two layers of $\mathrm{SiO}_{2}$ particles, a layer of nanophosphors of the exact same thickness than that deposited to build the resonators.

In order to realize the measurements required for our work, the set up presented in Fig. 1 was disposed. A green solid-state laser emitting at $\lambda=532 \mathrm{~nm}$ is used as a continuous wave excitation source. A $30 \mathrm{~mW}$ beam is normally incident to the sample surface, focused down by means of a 10 $\mathrm{cm}$-focal length lens. The light coming out of the multilayer structure in transmission mode is collected by an objective with a numerical aperture N.A. $=0.6$, coupled into a multimode fiber, and spectrally dispersed by a monochromator

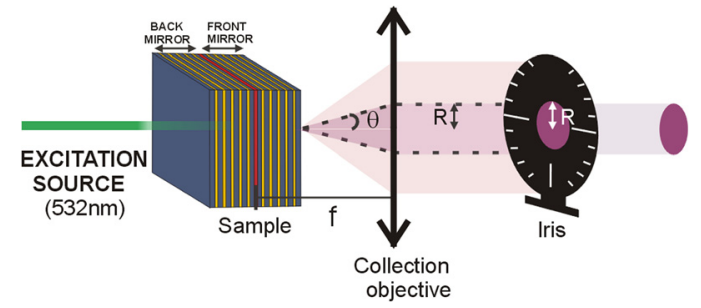

FIG. 1. (Color online) Experimental set-up employed for the angular characterization for the emission. The source represented is the $532 \mathrm{~nm}$ laser and the sample is the resonator made of nanoparticles of $\mathrm{SiO}_{2}$ (dark layers) and $\mathrm{TiO}_{2}$ (light layers). The middle layer represents the nanophosphor layer. 


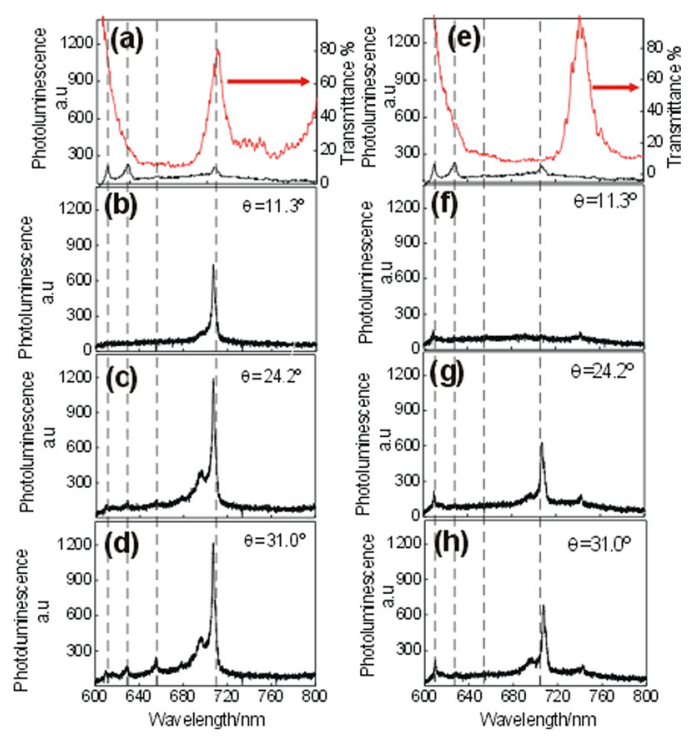

FIG. 2. (Color online) Transmittance (light lines) and photoluminescence (dark lines) spectra of optical resonators whose cavity modes are centered at $\lambda_{\mathrm{c}}=708 \mathrm{~nm}$ (a) and at $\lambda_{\mathrm{c}}=742 \mathrm{~nm}$ (e). Their photoluminescence spectra collected at different angles are plotted in sets of graphs b, c, d and f, g, h, respectively. Vertical dashed lines indicate the position of the emission bands of the nanophosphors.

attached to a charge-coupled device (CCD) detector camera. An iris is placed between the objective and the detector to study the relation between the intensity of the emission bands and the light cones of angular widths comprised between $5.7^{\circ}$ and $36.8^{\circ}$. The collection angle $\theta$ is determined through the radio $\mathrm{R}$ of the iris according to $\operatorname{tg}(\theta)=\mathrm{R} / \mathrm{f}$, where $\mathrm{f}$ is the focal of the objective $(\mathrm{f}=5 \mathrm{~mm})$. Modes that can be extracted from the structure are those whose internal emission angle $\alpha, \alpha=\sin ^{-1}\left(\sin (\theta) \mathrm{n}_{\mathrm{air}} / \mathrm{n}_{\mathrm{c}}\right)$, is smaller than the critical angle $\alpha_{\mathrm{c}} \sim 52^{\circ}$; above $\alpha_{\mathrm{c}}$, modes remain guided, i.e., they do not radiate to air. On the other hand, modes can be detected provided the collection angle matches the N.A. of the objective, $\theta<37^{\circ}$. The transmittance spectra were obtained using the same set up but using a white supercontinuum laser that allows us to measure optical spectra between $400 \mathrm{~nm}$ and $800 \mathrm{~nm}$.

Transmittance and photo-emission spectra corresponding to resonators with different cavity modes, namely $\lambda_{\mathrm{c}}=708 \mathrm{~nm}$ and $\lambda_{\mathrm{c}}=742 \mathrm{~nm}$, are plotted in Fig. 2 (left and right panels, respectively). The transmittance spectra (light lines) as well as the luminescent emission spectrum of the reference (dark lines) are shown in Figs. 2(a) and 2(e). The peaks observed in the transmittance spectra correspond to the transmitting window opened due to the presence of a resonant mode. In one case, the resonance coincides with the emission band at $\lambda_{\mathrm{c}}=708 \mathrm{~nm}$ (on-resonance), while in the other it does not match any luminescence line (off-resonance). The two sets of Figs. 2(b)-2(d) and 2(f)-2(h) display the photoluminescence emitted by the nanophosphors embedded in each type of resonator, respectively. For small angles of collection, the luminescent band at $\lambda_{\mathrm{B}}=708 \mathrm{~nm}$ is strongly enhanced when it is on-resonance, Fig. 2(b), and it is almost totally attenuated when it is off-resonance, Fig. 2(f). For the first case, it is observed that the intensity of the band at $\lambda_{\mathrm{B}}=708 \mathrm{~nm}$ increases, as the iris is gradually opened, until it reaches a constant value. The angular disper-
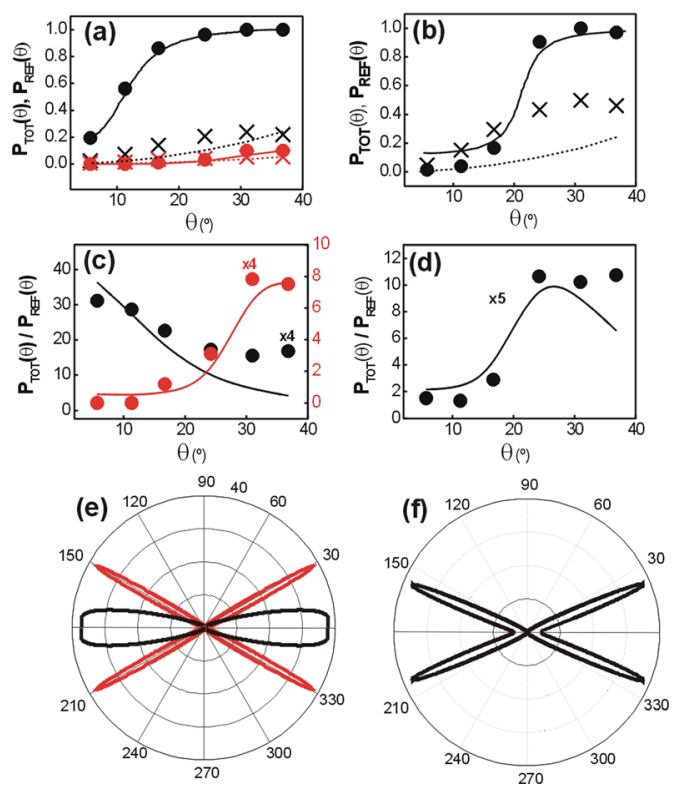

FIG. 3. (Color online) (a) Extraction power in an escape cone of angle $\theta$ of emission bands centered at $\lambda_{\mathrm{B}}=708 \mathrm{~nm}$ and $\lambda_{\mathrm{B}}=655 \mathrm{~nm}$ measured from the resonator whose cavity mode is located at $\lambda_{\mathrm{C}}=708 \mathrm{~nm}\left(P_{\text {TOT }}(\theta)\right.$, dark and light dots, respectively) and for reference layers $\left(P_{\text {ref }}(\theta)\right.$, dark and light crosses, respectively). (b) Similar plot of $P_{T O T}(\theta)$ and $P_{\text {ref }}(\theta)$ vs $\theta$ from a resonator characterized by $\lambda_{\mathrm{C}}=742 \mathrm{~nm}$. Graphs in (c) and (d) show the experimental enhancement factors $\left(P_{T O T}(\theta) / P_{\text {ref }}(\theta)\right)$ for these two resonators, respectively, using the same code to identify bands. Experimental curves are multiplied by a factor 4 and 5 for the resonator of (a) and (b), respectively. Theoretical curves obtained from Eq. (3) are plotted in Figs. 3(a)-3(d) as solid lines for both resonators and as dotted lines for references. Finally, (e) and (f) show the corresponding calculated polar emission patterns of bands $\lambda_{\mathrm{B}}=708 \mathrm{~nm}$ (dark lobes) and $\lambda_{\mathrm{B}}=655 \mathrm{~nm}$ (light lobes) as a function of the collection angle.

sion of the extracted power stabilizes at a larger angle in the case of the second resonator. Since the spectral position of the resonance moves towards shorter wavelengths as the angle of emission increases with respect to the normal to the multilayer surface, above a certain collection angle $\theta$, it will eventually match the position of an emission band, whose intensity is then enhanced. Thus, the cavity favors the extraction of different wavelengths at different angles.

In Fig. 3, we plot the experimentally observed evolution of the intensities of the emission bands affected by the cavity resonances versus collection angle for the two resonators considered, with $\lambda_{\mathrm{c}}=708 \mathrm{~nm}$ (Fig. 3(a)) and $\lambda_{\mathrm{c}}=742 \mathrm{~nm}$ (Fig. 3(b)). The intensities of all the emission bands were divided by the maximum value measured for the band at $\lambda_{\mathrm{c}}=708 \mathrm{~nm}$ for the sake of clarity and to facilitate the comparison with the theoretical results. In the first case, the normalized intensities of bands placed at $\lambda_{\mathrm{B}}=655 \mathrm{~nm}$ (light circles) and $\lambda_{\mathrm{B}}=708 \mathrm{~nm}$ (dark circles) are plotted, while in the second case only the results for the band at $\lambda_{\mathrm{B}}=708 \mathrm{~nm}$ are drawn, since only this band matches the resonant mode at a certain angle among those considered. The angular variation of the intensity of the peaks emitted by the layer of nanophosphors used as reference is plotted for the sake of comparison (dark and light crosses for $\lambda_{\mathrm{B}}=708 \mathrm{~nm}$ and $\lambda_{\mathrm{B}}=655 \mathrm{~nm}$, respectively). The information for the rest of the bands is omitted for clarity.

An analysis of these angular trends was performed by comparing them to the emission of a dipole placed in the 
center of a perfectly uniform planar microcavity. This system was analyzed theoretically by Benisty et al. ${ }^{14}$ In that study, an expression that relates the power extracted out of the resonator, $P_{\text {out }}$, in a specific direction $\theta$ is derived

$$
P_{\text {out }}(\theta) \propto \frac{2 T_{1}}{\left|1-r_{1} r_{2} e^{2 i \phi}\right|^{2}} \frac{1+r_{2}^{2}-2 r_{2} \cos \left(2 \phi^{\prime}\right)}{2},
$$

where $L$ is the thickness of the cavity $\left(L=\lambda_{c} / 2 n_{c}\right), k$ is the wavenumber of the emitted light inside the cavity at a wavelength $\lambda_{\mathrm{B}}\left(\mathrm{k}=2 \pi \mathrm{n}_{\mathrm{C}} / \lambda_{\mathrm{B}}\right), r_{1}$ and $r_{2}$ are the amplitude reflectivities of the fields on the front and back mirrors, respectively, $T_{1}=1-r_{1}^{2}$ is the front mirror transmission, $\phi^{\prime}=k z \cos \alpha, z$ being the distance from the emitter to the back mirror (considered $\mathrm{z}=\mathrm{L} / 2$ ), and $\phi=k L \cos \alpha$.

Thus, the total power escaping from the resonator and collected within a cone of angular width $\theta$ is given by

$$
P_{T O T}(\theta) \propto 2 \pi \int_{0}^{\theta} P_{\text {out }}(\theta) \cdot \sin \theta \cdot d \theta .
$$

Our input parameters are the spectral position of the luminescent band, $\lambda_{B}$, and the finesse of the resonance, $F$, extracted from the measured spectra. The coefficients $r_{1}$ and $r_{2}$ are estimated from $F$ through the expression

$$
F=\frac{\pi \sqrt{r_{1} r_{2}}}{1-r_{1} r_{2}} .
$$

The calculated trends for $P_{T O T}(\theta)$ versus $\theta$, obtained from Eq. (2), are plotted as solid lines in Figs. 3(a) and 3(b) for the chosen bands, and as dashed lines for homogeneous media (reference layers). It can be seen that these are in good qualitative agreement with the experimental ones in all cases. It should be noted that, since the model does not take into account the intensities of the different emission bands of the nanophosphors of the reference, only qualitative information can be extracted from the evolution of the curve. All calculated curves shown in Figs. 3(a) and 3(b) are drawn so that the relation between the intensities of the different bands observed experimentally is preserved.

The monotonic growth of the intensity emitted at $\lambda_{\mathrm{B}}=708 \mathrm{~nm}$ presents a very different angular dependence as a function of the relative position of $\lambda_{\mathrm{C}}$ and $\lambda_{\mathrm{B}}$. In Figs. 3(c) and $3(\mathrm{~d})$, the experimental and theoretical angular dependences of the emission enhancement, estimated as the ratio between the photoluminescence power extracted at a certain collection angle $\theta$ from the resonator and that collected from the reference samples, $P_{T O T}(\theta) / P_{\text {ref }}(\theta)$, are shown. It can be seen that the theoretical trends (dark and light solid lines in Figs. 3(c) and 3(d)) are, again, in qualitative agreement with the measurements (dark and light circles in Figs. 3(c) and 3(d)).

Quantitatively, however, experimental and theoretical data differ by a factor comprised between 4 and 5 . Thus, experimental curves are multiplied by a factor 4 and 5 for the resonator of Figs. 3(a) and 3(b), respectively. The differences observed are very likely due to the deviations of the experimental structure from the one considered theoretically. The main differences arise from the fluctuations of the thickness of the optical cavity as a result of the roughness introduced by the silica nanoparticles, whose average size is around 30 $\mathrm{nm}$. Also, the emitters are not exactly placed in the middle of the cavity as assumed in the theoretical model, since the thickness of the layer of rare-earth based particles sandwiched between silica layers represents approximately $10 \%$ of the total thickness of the cavity. In addition, both polarization effects and the real multilayer structure of the cavity mirrors have been neglected in the model. Remarkably, in spite of all these simplifications, the model solutions nicely reproduce the main observed features. Finally, theoretical polar emission patterns are shown in Figs. 3(e) and 3(f) to further illustrate the effects herein discussed. Very different angular distribution of energy for the bands emitted at 708 $\mathrm{nm}$ and $655 \mathrm{~nm}$ are attained as a function of the resonator in which the nanophosphors are integrated. These observations are in good agreement with both experimental and theoretical studies previously realized for quantum wells enclosed by a one-dimensional photonic crystal microcavity. ${ }^{18}$

In conclusion, the angular properties of light emitted from nanophosphors embedded in optical resonators built in one-dimensional photonic crystal coatings have been investigated. Abrupt angular variations of the enhancement caused by the photonic structure and the extraction power are observed, which are in qualitative agreement with the solutions of a simple theoretical model for light emitted by a dipole inside a perfect microcavity. Our results demonstrate that integration of nanophosphors within photonic crystals provides ways of tailoring the emission properties that could be put into practice in phosphorescent displays.

We acknowledge the Spanish Ministry of Science and Innovation for funding provided under grants MAT200802166 and CONSOLIDER HOPE CSD2007-00007, to Junta de Andalucía for grants FQM3579 and FQM5247. M.E.C. acknowledges the Junta de Andalucía for funding of his contract. These results are within the scope of C'Nano IdF and were partially supported by the Région Ile-de-France. C'Nano IdF is a CNRS, CEA, MESR, and Région Ile-deFrance Nanosciences Competence Center.

${ }^{1}$ V. L. Colvin, M. C. Schlamp, and A. P. Alivisatos, Nature 370, 354 (1994).

${ }^{2}$ I. L. Medintz, H. T. Uyeda, E. R. Goldman et al., Nature Mater. 4, 435 (2005).

${ }^{3}$ R. Roshan, P. N. Taylor, and D. J. Montgomery, Patent Application GB20060019587 "A display with a primary light source for illuminating a nanophosphor re-emission material", priority date 04/10/2006.

${ }^{4}$ W. S. Song, H. N. Choi, Y. S. Kim et al., J. Mater. Chem. 20, 6929 (2010).

${ }^{5}$ G. R. S. Raju, H. C. Jung, J. Y. Park et al., J. Optoelectron. Adv. Mater. 12(6), 1273 (2010).

${ }^{6}$ X. Yu and Y. Wang, J. Alloys Compd. 497, 290 (2010).

${ }^{7}$ G. R. S. Raju, H. C. Jung, J. Y. Park et al., J. Alloys Compd. 481, 730 (2009).

${ }^{8}$ G. S. R. Raju, J. Y. Park, H. C. Jung et al., Opt. Mater. 31, 1210 (2009).

${ }^{9}$ X. Qin, Y. Ju, S. Bernhard et al., Mater. Res. Bull. 42, 1440 (2007).

${ }^{10}$ O. Sanchez-Sobrado, M. E. Calvo, N. Nuñez et al., Nanoscale 2, 936 (2010).

${ }^{11}$ N. Ganesh, I. D. Block, P. C. Mathias et al., Opt. Express 16, 21626 (2008).

${ }^{12}$ C. H. Chao, H. C. Kuo, and W. Y. Lee, Appl. Phys. Lett. 94, 123106 (2009).

${ }^{13}$ C. F. Lai, H. C. Kuo, P. Yu et al., Appl. Phys. Lett. 97, 013108 (2010).

${ }^{14}$ H. Benisty, H. De Neve, and C. Weisbuch, IEEE J. Quantum Electron. 34, 9 (1998).

${ }^{15}$ N. O. Nuñez and M. Ocaña, Nanotechnology 18, 455606 (2007).

${ }^{16}$ S. Colodrero, M. Ocaña, and H. Míguez, Langmuir 24, 4430 (2008).

${ }^{17}$ M. E. Calvo, O. Sánchez-Sobrado, S. Colodrero et al., Langmuir 25, 2443 (2009).

${ }^{18}$ G. Bjork, S. Machida, Y. Yamamoto et al., Phys. Rev. A 44, 669 (1991). 